\title{
Erratum: General relations between the power, efficiency, and dissipation for the irreversible heat engines in the nonlinear response regime [Phys. Rev. E 97, 012141 (2018)]
}

\author{
I. Iyyappan and M. Ponmurugan
}

(Received 21 August 2019; published 3 September 2019)

DOI: 10.1103/PhysRevE.100.039901

We would like to point out a few of the equations related to minimum Dissipation $(\mathrm{mD})$ were incorrect in the published version of the paper. However, these errors do not affect the main results of the article. The corrected equations and the corresponding Figs. 3 and 5 are given below:

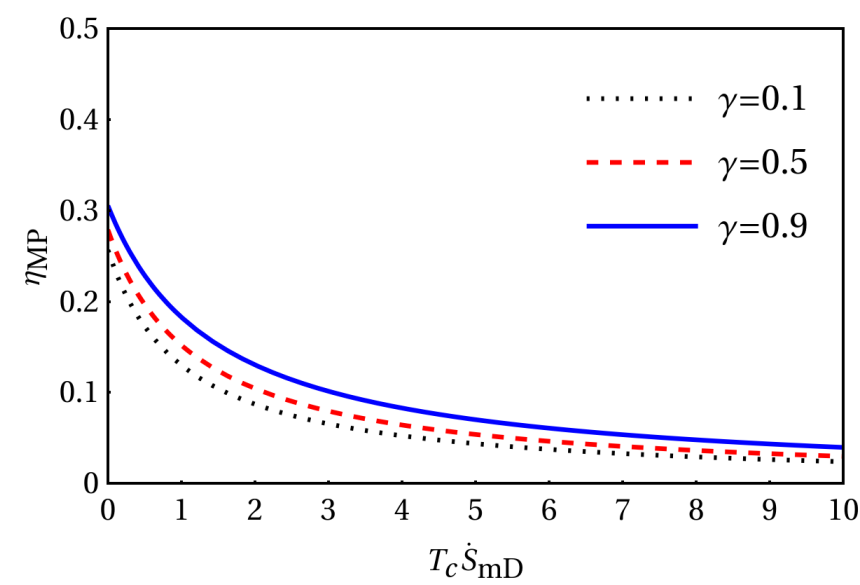

FIG. 3. The efficiency at maximum power (MP) [obtained from Eq. (28)] is plotted as a function of the minimum dissipation for different values of $\gamma=0.1$ for the dotted curve, $\gamma=0.5$ for the dashed curve, and $\gamma=0.9$ for the solid curve. Here we set $\eta_{C}=0.5, \eta_{\mathrm{ME}}=0.45, P_{\mathrm{MP}}=$ 0.5 , and $P_{\mathrm{ME}}=0.1$ (where ME stands for maximum efficiency).

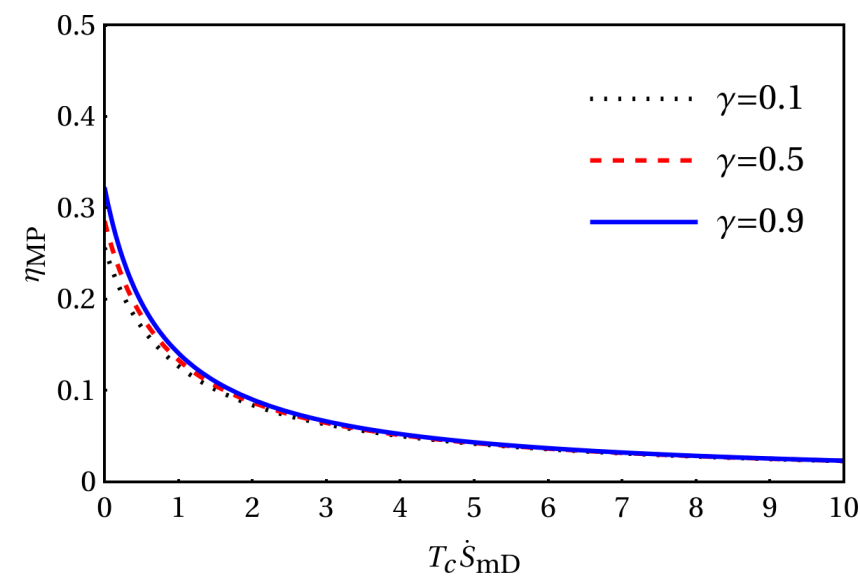

FIG. 5. The efficiency at maximum power from Eq. (32) is plotted as a function of the dissipation for different values of $\gamma$, where we have $\gamma=0.1$ for the dotted curve, $\gamma=0.5$ for the dashed curve, and $\gamma=0.9$ for the solid curve. Here we set $\eta_{C}=1$ and $P_{\mathrm{MP}}=1$. 


$$
\begin{aligned}
T \dot{S}_{m D} & =\left(\frac{\eta_{C}}{\eta_{\mathrm{MP}}}-\frac{\eta_{C}^{2}}{\eta_{\mathrm{ME}}^{2}}-1\right) P_{\mathrm{MP}}+\frac{\eta_{C}^{2}}{\eta_{\mathrm{ME}}^{2}} P_{\mathrm{ME}} . \\
P_{m D} & =0, \quad T \dot{S}_{m D}=P_{\mathrm{MP}}\left(\frac{\eta_{C}}{\eta_{\mathrm{MP}}}-2\right) . \\
J_{3} & =\frac{L_{21}-L_{12} \eta_{C}}{L_{11}} J_{1}+\frac{\mathcal{D}}{L_{11}} X_{2}+\gamma_{c} J_{1}^{2} . \\
\dot{S}_{m D} & =-\frac{\left[L_{12}^{2}+L_{21}^{2}+4 \gamma \eta_{C} \mathcal{D}-4 \mathcal{D}-2 L_{12} L_{21}\right]}{4 L_{11}\left(1-\gamma \eta_{C}\right)} X_{2}^{2} . \\
P_{m D} & =\eta_{C} \frac{\left(L_{12}-L_{21}\right)\left[L_{12}\left(1-2 \gamma \eta_{C}\right)+L_{21}\right]}{4 L_{11}\left(1-\gamma \eta_{C}\right)^{2}} X_{2} . \\
P_{m D} & =0, \quad T_{c} \dot{S}_{m D}=\left(\frac{\eta_{C}}{\eta_{\mathrm{MP}}}-2+\gamma \eta_{C}\right) P_{\mathrm{MP}} . \\
P_{m D} & =0, \quad\left(T_{c} \dot{S}_{m D}\right)^{\mathrm{sym}}=P_{\mathrm{MP}}\left(\frac{\eta_{C}}{\eta_{\mathrm{MP}}}-2+\frac{\eta_{C}}{2}\right) . \\
\left.\dot{S}_{m D}\right)^{-} & =\left(\frac{\eta_{C}}{\eta_{\mathrm{MP}}}-\frac{\eta_{C}^{2}}{\eta_{\mathrm{ME}}^{2}}-1\right) P_{\mathrm{MP}}+\frac{\eta_{C}^{2}}{\eta_{\mathrm{ME}}^{2}} P_{\mathrm{ME}} . \\
P_{m D} & =0, \quad\left(T_{c} \dot{S}_{m D}\right)^{-}=P_{\mathrm{MP}}\left(\frac{\eta_{C}}{\eta_{\mathrm{MP}}}-2\right) . \\
\eta_{\mathrm{MP}} & \left.\dot{S}_{m D}\right)^{+}=P_{\mathrm{MP}}\left(\frac{\eta_{C}}{\eta_{m}}-2+\eta_{C}\right) .
\end{aligned}
$$

Furthermore, the minimum dissipation relations Eqs. (28), (40), and (46) should be read as

$$
\begin{aligned}
T_{c} \dot{S}_{m D} & =\left[\frac{\eta_{C}}{\left(1-\gamma \eta_{C}\right) \eta_{\mathrm{MP}}}-\frac{\eta_{C}^{2}}{\left(1-\gamma \eta_{C}\right) \eta_{\mathrm{ME}}^{2}}-1\right] P_{\mathrm{MP}}+\frac{\left(1-\gamma \eta_{\mathrm{ME}}\right)}{\left(1-\gamma \eta_{C}\right)} \frac{\eta_{C}^{2}}{\eta_{\mathrm{ME}}^{2}} P_{\mathrm{ME}} . \\
\left(T_{c} \dot{S}_{m D}\right)^{+} & =\frac{1}{\left(1-\eta_{C}\right)}\left(\frac{\eta_{C}}{\eta_{\mathrm{MP}}}-\frac{\eta_{C}^{2}}{\eta_{\mathrm{ME}}^{2}}-1+\eta_{C}\right) P_{\mathrm{MP}}+\frac{\left(1-\eta_{\mathrm{ME}}\right)}{\left(1-\eta_{C}\right)} \frac{\eta_{C}^{2}}{\eta_{\mathrm{ME}}^{2}} P_{\mathrm{ME}} . \\
\left(T_{c} \dot{S}_{m D}\right)^{\mathrm{sym}} & =\left[\frac{2 \eta_{C}}{\left(2-\eta_{C}\right) \eta_{\mathrm{MP}}}-\frac{2 \eta_{C}^{2}}{\left(2-\eta_{C}\right) \eta_{\mathrm{ME}}^{2}}-1\right] P_{\mathrm{MP}}+\frac{\left(2-\eta_{\mathrm{ME}}\right)}{\left(2-\eta_{C}\right)} \frac{\eta_{C}^{2}}{\eta_{\mathrm{ME}}^{2}} P_{\mathrm{ME}} .
\end{aligned}
$$

